\title{
Síndrome de restauración inmune asociado a tratamiento antirretroviral y criptococosis meníngea. Caso clínico
}

\author{
David Sáez $M^{1,2}$, Laura Bahamondes $M^{3}$, \\ Gislaine Lam E ${ }^{1,2}$, Luz Arellano $R^{2}$, Patricia Lillo $Z^{1,2}$. \\ Inflammatory reconstitution immune \\ syndrome associated to antiretroviral \\ therapy and meningeal \\ cryptococcosis
}

The objective of high activity antiretroviral therapy (HAART) in patients with AIDS, is to obtain immune restoration. This means a reduction of the viral load and restitution of the $\mathrm{CD}_{4}$ cell count. A decreased rate of HIV replication improves both the number and function of $\mathrm{CD}_{4}$ cells. Nevertheless, this treatment sometimes results in the reappearance of previous symptoms from treated conditions due to opportunistic infections (ie: tuberculosis, criptococcosis, hepatitis, Pneumocystis jirovesi, toxoplasmosis, etc) or non infectious condition such as sarcoidosis, Graves disease or Kaposi sarcoma. This is known as Inflammatory Reconstitution Immune Syndrome (IRIS). We report a 37 year-old woman in stage C3-AIDS with a previous criptococcal meningitis. She was treated, achieving a marked improvement with treatment and subsequent suppressive therapy with fluconazole $200 \mathrm{mg} / \mathrm{day}$. IRIS appeared after 8 months of ongoing antiretroviral therapy with immune restoration with the development of aseptic meningitis and intracranial hypertension. The opportunistic agent could not be identified by cultures. Additional laboratory tests excluded toxoplasmosis, tuberculosis, bacterial cerebral abscesses, syphilitic cerebral gummas, and lymphoma. Brain CT and magnetic resonance studies were compatible with brain vasculitis and leptomeningitis. The patient condition improved with general measures, such as a repeated lumbar punctures and non steroidal anti-inflammatory drugs. We conclude that this patient had an IRIS due to a Cryptococcus neoformans antigen (Rev Méd Chile 2006; 134: 1310-14).

(Key words: Acquired immunodeficiency syndrome; Antiretroviral therapy, highly active; Cryptococcus neoformans)

Recibido el 18 de octubre, 2005. Aceptado el 14 de marzo, 2006.

${ }^{1}$ Servicio de Neurología, Hospital Barros Luco-Trudeau. ${ }^{2}$ Departamento de Neurología Campus Sur, Universidad de Chile. ${ }^{3}$ Hospital de Enfermedades Infecciosas $\$ D r . ~ L u c i o$ Córdova» Santiago de Chile.

Correspondencia a: Dr. David Sáez Méndez. Departamento de Neurología Campus Sur, Universidad de Chile. Gran Avenida J.M. Carrera 3204, San Miguel, Santiago de Chile. Fono-Fax: 562 3948941. E mail: dsaez@med.uchile.cl 
El objetivo del tratamiento antirretroviral en los pacientes con infección por VIH/SIDA es la mejoría de la capacidad de respuesta inmunológica. Esto se ha conseguido con la terapia antirretroviral de gran efectividad (TARGA), con la cual hay un reestablecimiento de la capacidad de respuesta inmune celular, evidenciable por el aumento de los CD4+ y la disminución de la carga viral. Sin embargo, en ocasiones, esta respuesta resulta en el deterioro clínico del paciente, efecto paradojal que se observa especialmente en aquellos que han presentado una infección oportunista previa. Esta respuesta conocida como síndrome inflamatorio de restauración inmune» (SIRI) es especialmente grave cuando ocurre a nivel del sistema nervioso central ${ }^{1,2}$.

Presentamos el caso de una paciente con antecedentes de VIH/SIDA, criptococosis en terapia de supresión crónica, que en relación a instauración del TARGA, presentó una reactivación del cuadro clínico, compatible con una recidiva de su meningitis por criptococo.

\section{CASO CĹNICO}

Paciente de sexo femenino de 37 años, con infección por VIH en etapa C 3 (según clasificación CDC 1993), diagnosticada en 2003, cuando presentó una neumonía por Pneumocystis jirovesi. En marzo de 2004 presentó meningitis criptocócica caracterizada por cefalea, compromiso cualitativo de conciencia, fiebre y signología meníngea. El estudio de líquido cefalorraquídeo (LCR) demostró proteínas de 5,68 g/l, leucocitos 5/campo, $100 \%$ mononucleares, glucosa de $30 \mathrm{mg} / \mathrm{dl}$ (glicemia concomitante $98 \mathrm{mg} / \mathrm{dl}$ ), examen directo por tinta china positivo, en la tinción de Gram se observaron levaduras 2 cruces, con yemación y pseudohifas. Hemocultivo positivo para hongos (levaduras en gran cantidad). Cultivo de LCR positivo para criptococo neoformans. Inmunofluorescencia (IFI) para toxoplasmosis: negativa, ADA 12 U/l, baciloscopia negativa. Tomografía computada (TC) encefálica mostró hipodensidad de núcleo caudado bilateral, interpretada como lesión vascular.

Se trató con anfotericina B $40 \mathrm{mg}$ al día por 14 días (560 mg en total). La paciente evolucionó de manera satisfactoria, continuó con fluconazol 400 mg vo cada $12 \mathrm{~h}$ y sulfametoxazol/trimetoprima (800 mg/160 mg) 1 comprimido al día. Cultivo para hongos negativo en LCR a los 15 días, pero con tinta china positivo. Carga viral (15.04.04): copias 11.000, logaritmo 4 (copias/ml). CD4+ indetectable.

En forma ambulatoria, en abril de 2004, se inició terapia antirretroviral con efavirens $600 \mathrm{mg}$ en la noche, lamivudina $150 \mathrm{mg}$ cada $12 \mathrm{~h} \mathrm{y}$ stavudina $30 \mathrm{mg}$ cada $12 \mathrm{~h}$ vo.

Posteriormente, el 21.05.04 reingresó por sospecha de reactivación de meningitis por criptococo por cefalea intensa, alza térmica, compromiso del estado general de una semana de evolución, signología meníngea, sin focalidad neurológica ni compromiso de conciencia. TC cerebral sin contraste, sin nuevas lesiones. LCR evidenció, proteinorraquia: 1,14 g/l, glucosa 0,41 mg/dl, 14 leucocitos por campo, 100\% mononuclear, cultivo negativo, tinta china con levaduras capsuladas. ADA 8,4 U/L y baciloscopia negativa. Cultivo LCR negativo para hongos y Koch. VDRL negativo. Se indicó anfotericina B $35 \mathrm{mg}$ al día por 14 días (490 mg en total), profilaxis con sulfametoxazol/trimetoprima ( $800 \mathrm{mg} / 160 \mathrm{mg}$ ) un comprimido al día. Permaneció asintomática hasta mayo de 2005, cuando fue hospitalizada por cuadro de 6 días de evolución, caractenzado por cefalea holocránea leve a moderada, náuseas, confusión simple y rigidez de nuca, sin fiebre. Se solicitó TC de cerebro, que informó lesiones compatibles con vasculitis (Figura 1). El estudio de LCR mostró líquido claro, transparente, con goteo aumentado, glucorraquia $45 \mathrm{mg} / \mathrm{dl}$ (glicemia $78 \mathrm{mg} / \mathrm{dl}$ ), proteinorraquia 0,55 g/l, leucocitos 5 por campo, 100\% mononucleares, cultivo bacteriano negativo, cultivo para hongos negativo a los 15 días, tinta china negativo, baciloscopia negativa, ADA 4,4 U/l, hemocultivos negativos. Continuó en similares condiciones, pero con desaparición de signología meníngea. Se repitió punción lumbar que dio salida a LCR claro, transparente, con goteo aumentado, glucorraquia: $60 \mathrm{mg} / \mathrm{dl}$, proteinomaquia $0,24 \mathrm{~g} / \mathrm{l}$, VDRL no reactivo, leucocitos $5 / \mathrm{mm}^{3}$ $100 \%$ mononucleares, tinta china y cultivo para hongos negativos, ADA 4,4 U/L: Linfocitos: CD4+ $121 / \mathrm{mm}^{3}$ (14.06.05) Carga viral indetectable. Se mantuvo tratamiento con TARGA. Resonancia nuclear magnética (RM) encefálica informó reforzamiento leptomeníngeo y lesiones compatibles con 


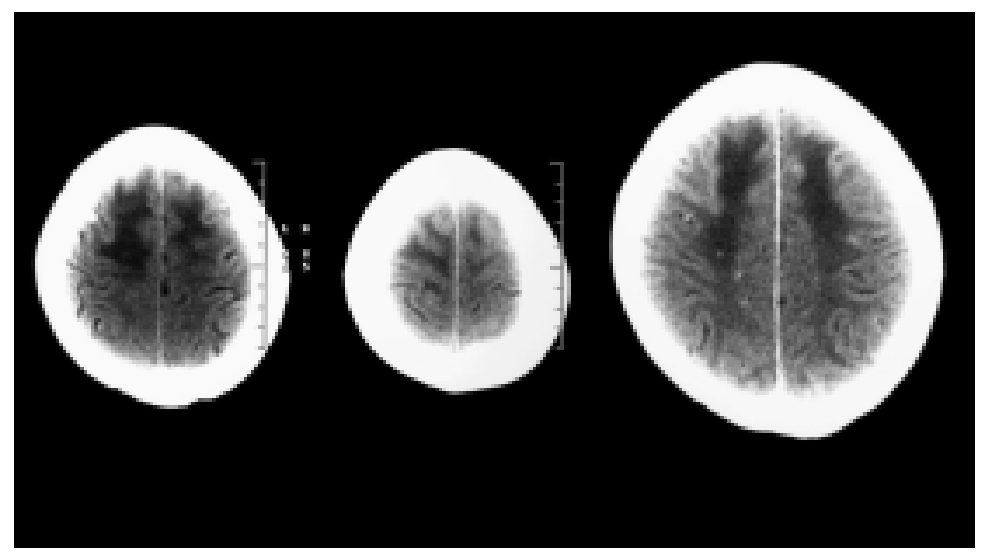

FIguRA 1. A. Imagen de TC cerebral sin contraste muestra zonas hipodensas en sustancia blanca frontal bilateral; B. Imagen de TC con contraste que muestra iguales hallazgos, además de pequeñas zonas de refuerzo nodular.

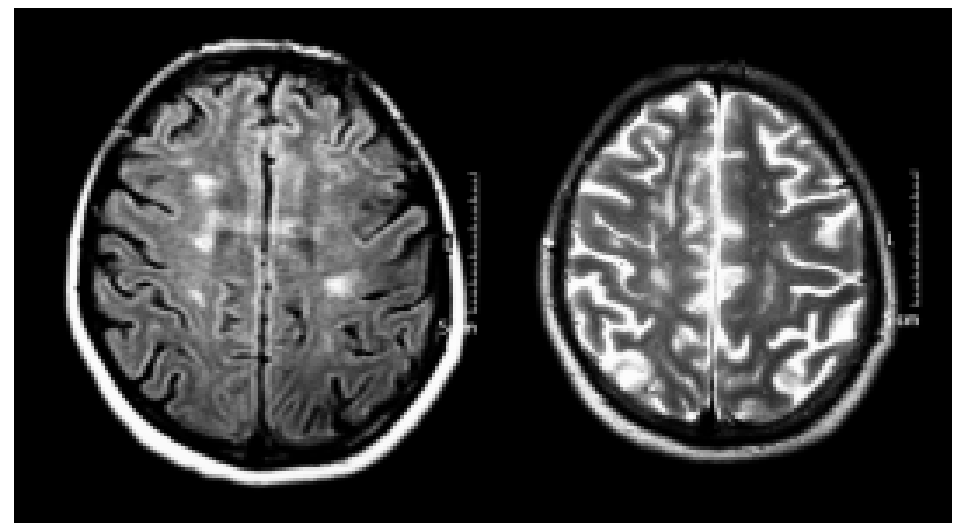

FIgURA 2. A. RM cerebral (T1 Gad) muestra imagen hipointensa en región frontal bilateral además de áreas de captación de Gadolinio, señalando un defecto de barrera hematoencefálica. B. RM cerebral (T2), muestra imagen hiperintensa en ambas regiones frontales en relación a áreas hipointensas del T1, las zonas captantes de Gadolinio en el T1, se aprecian espontáneamente hiperintensas en esta secuencia.

criptococomas y reacción inflamatoria (Figura 2). Por el cuadro clínico, apoyado en los hallazgos imagenológicos y en la recuperación inmunológica, se planteó el diagnóstico de síndrome inflamatorio de reinstauración inmune asociado a criptocococis meníngea. Se realizó tratamiento de tipo sintomático, con antiinflamatorios no esteroidales y punciones lumbares evacuadoras de líquido cefalorraquídeo. Con este tratamiento la paciente presentó una progresiva mejonía clínica, encontrándose 3 meses después (septiembre de 2005) asintomática de su cuadro neurológico.

\section{Discusión}

Las manifestaciones del HIV en el sistema nervioso central son variadas, desde un compromiso mínimo hasta severos trastornos capaces de llevar a la muerte al paciente ${ }^{3-6}$.
La criptococosis misma es en sí pleomórfica, lo habitual es que la meningitis criptocócica se produzca en severos estados de inmunodepresión de tipo celular y con un bajo recuento de linfocitos CD4+. Diagnosticada y tratada oportunamente, se obtiene la remisión del cuadro, requiriendo posteriormente terapia antimicótica de supresión crónica. Sin embargo, la mortalidad de la meningitis criptocócica alcanza a 30\% a 40\%, lo que ocurre principalmente en las primeras semanas de tratamiento ${ }^{3,7}$.

El SIRI, fue descrito en relación a una agravación de un cuadro de linfadenitis por complejo Micobacterium avium (MAC), concomitantemente con la recuperación de capacidad de respuesta inmune, en pacientes con SIDA en tratamiento ${ }^{1,8,9}$. Posteriormente, estos cuadros han sido reportados igualmente en otras patologías del SIDA, siempre asociados a la triterapia y en otras condiciones, como esclerosis múltiple ${ }^{10}$. Si bien la 
TARGA ha llevado a una dramática reducción en los casos generales y neurológicos asociados al SIDA, en esta etapa, han aparecido episodios de reactivación de cuadros infecciosos previamente diagnosticados, o bien, que permanecían latentes en forma subclínica. Entre ellos, se han comunicado también casos de tuberculosis pulmonar, neumonía por Pneumocystis jirovesi, infecciones por virus herpes, citomegalovirus, hepatitis B y C, infecciones por criptococo neoformans, y además otras alteraciones autoinmunes. En el sistema nervioso central se han comunicado reactivaciones de meningitis tuberculosa, tuberculomas, meningitis criptocócica y criptococomas, toxoplasmosis, leucoencefalopatía multifocal progresiva por virus JC e infecciones virales como citomegalovirus y herpes. A nivel vascular cerebral, se ha notificado un caso de aneurismas múltiples como parte de este síndrome y en lo periférico, un síndrome de Guillain Barre-símil ${ }^{1,11-}$ 16. La incidencia del SIRI, en general, es de alrededor de $30 \% 2$, y para la criptococosis la incidencia es de 4,2 por 100 personas/año, en pacientes que habían presentado una infección previa por este microorganismo ${ }^{17}$, produciéndose, en general, en los primeros 8 meses de instaurada la TARGA. Estos cuadros se apartan de los efectos tóxicos por los antirretrovirales que en general son más arrastrados, como por ejemplo: depresión, alteraciones leves de la marcha, pérdida del control motor fino, síndromes cerebelosos y claramente la polineuropatía tóxica ${ }^{18}$. La TARGA puede producir daño en el corto plazo, lo que ocurre luego de días o semanas de iniciado o de cambio de régimen terapéutico, manifestándose como manía, psicosis, alteraciones de sustancia blanca, encefalopatía. Los mecanismos de ellas son desco-

\section{REFERENCIAS}

1. Shelburne $\mathrm{S}$, Hamll $R$. The immune reconstitution inflamatory síndrome. AIDS Rev 2003; 5: 67-79.

2. Shelburne S, Visnegarwala F, Darcourt J, Giordano T, White A, Hamll R. Incidence and risk factors for immune reconstitution inflammatory syndrome during highly active antiretroviral therapy. AIDS 2005; 19: 399-406. nocidos. A largo plazo, la toxicidad de la TARGA se asocia con un daño en el ADN mitocondrial y cambios en los niveles de lípidos de las células ${ }^{18}$.

El caso que relatamos trata de una paciente que presentó una meningitis por criptococo y que, estando en terapia de supresión crónica, presentó reactivación de su sintomatología, con TC y RM que mostraron lesiones compatibles con criptococomas y meningitis por criptococo, pero con una reacción inflamatoria vecina que no correspondía a la descripción típica de estos cuadros. Esto ocurrió en concomitancia a la recuperación de los $\mathrm{CD} 4+\mathrm{y}$ resultando el examen directo y cultivos repetidos en LCR para criptococo negativo. El tratamiento fue de tipo sintomático con analgésicos y punciones evacuadoras de LCR. Entre las medidas recomendadas para este cuadro está el uso de antiinflamatorios no esteroidales, diuréticos inhibidores de la anhidrasa carbónica, corticoides, diuréticos osmóticos, ventilación mecánica, punciones evacuadoras y derivaciones ventriculares, las que deben evaluarse según la necesidad en cada paciente.

En este caso hemos planteado el diagnóstico de síndrome inflamatorio de restauración inmune (SIRI) en respuesta a una criptococosis previa. El caso presentado cumple los tiempos reportados para casos de SIRI, la negatividad en la búsqueda del criptococo, la respuesta satisfactoria sin tratamiento antimicótico específico (sólo se mantuvo la terapia de supresión crónica con fluconazol 200 $\mathrm{mg} /$ día), presencia de signos de vasculitis en la imagenología; lo cual traduce una respuesta inflamatoria secundaria, y esencialmente la recuperación de la capacidad de respuesta inmune evidenciada por el aumento de los CD4+ y disminución de la carga viral.

3. Berger J, Simpson D. Neurologic complications os AIDS. En Infections of the central nervous system. Second Ed. Sheld W., Whitley R, Durack D. Eds. Lippincott-Raven Publishers 1997, Philadelphia pág 255-271.

4. Silva-Rosas C, Agar A, Guzmán MA, Thompson L, SEPúlveda C. Neurosífilis e infección por el virus de inmunodeficiencia humana (VIH-1). Rev Méd Chile 1994; 122: 1393-7. 
5. ClifFord DB. Human immunodeficiency virusassociated dementia. Arch Neurol 2000; 57: 321-4.

6. Silva-Rosas C, Afani A, Fruns M, Guzmán MA, SEPúlveda C. Meningitis en sujetos infectados con el virus de la inmunodeficiencia humana. Neurología (España) 1999; 14: 62-6.

7. Brouwer A, Rajanuwonn A, Chierakul W, GrifFing G, Larsen R, White N, Harrison T. Combination antifungal therapies for HIV-associated cryptococcal meningitis: a randomized trial. Lancet 2004; 363: 1764-7.

8. DiPerri G, Bonora S, Vento S, Aluegranzi B, Concia E. Highly active antiretroviral therapy (letter). Lancet 1998; 351: 1056.

9. Dworkin M, FratKin M. Mycobacterium avium complex lymph node abscess after use of highly antiretroviral therapy in a patient with Aids (letter). Arch Intern Med 1998; 158: 1828.

10. Langer-Gould A, Atias S, Bolien A, Peluetier D. Progressive multifocal leukoencephalopathy in a patient treated with natalizumab. $\mathrm{N}$ Engl J Med 2005; 353: 1-7 Published at www.nejm.org June 9, 2005 (10.1056/NEJMoa051847).

11. Shelborne S III, Hamil RJ, Rodríguez-Barradas MC, Greenberg SB, AtMAR RL, Musher DW et aL. Immune reconstitution inflammatory syndrome: emergence of a unique syndrome during highly active antiretroviral therapy. Medicine (Baltimore) 2002; 81: 213-27.
12. Collazos J, Mayo J, Martínez E, Bianco M-S. Contrastenhancing progressive multifocal leukoencephalopathy as an immune reconstitution event in AIDS patients. (letter). AIDS 1999; 13: 1426-8.

13. Mirales P, Berenguer J, Lacruz C, Cosin J, López J, PADILA B ET AL. Inflammatory reactions in progressive multifocal leukoencephalopathy after highly active antiretroviral therapy. AIDS 2001; 15: 1900-2.

14. Catteian A, Trevenzol M, Sasset L, Lanzafame M, Marchioro U, MeneghrtTi F. Multiple cerebral criptococomas associated with immune reconstitution in HIV-1 infection (letter). AIDS 2004; 18: 349-50.

15. LAWN S, BEKKER L, MiLeR R. Immune reconstitution disease associated with mycobacterial infections in HIV-infected individuals receiving antiretrovirals. Lancet Infect Dis 2005; 5: 361-73.

16. Belobel P, Brassat D, Delisle M, Scaravim F, Clanet M. Progressive multifocal leukoencephalopathy in a HIV patient with normal CD4 T-cell count and magnetic resonance imaging (letter). AIDS 2004; 18: 702-4.

17. Lortholary O, Fontanet A, Memain N, Martín A, SITBON K, DROMER F. Incidence and risk factors of immune reconstitution inflammatory síndrome complicating HIV-associated cryptococcosis in France. AIDS 2005; 19: 1043-9.

18. LANGFoRd D. Chronic exposure of the Blood-Brain Barrier to highly active antiretroviral therapy. Potencial consequences on the CNS. Infect Dis Clin Pract 2004; 12: 158-62. 